\title{
Protocolos de Terapia Cognitivo-Comportamental culturalmente adaptados para tratamento de Tept: uma revisão sistemática da literatura
}

\section{Cognitive-Behavioral Therapy protocols culturally adapted for PTSD Treatment: a systematic review of the literature}

\author{
Alice Einloft Brunnet (orcid.org/0000-0001-5290-1343) 1 \\ Laura Teixeira Bolaséll (orcid.org/0000-0001-9779-7842)² \\ Marcelo Klock Bujak (orcid.org/0000-0001-8685-8072)33 \\ Marcelo Montagner Rigoli (orcid.org/0000-0003-3721-5729)4 \\ Christian Haag Kristensen (orcid.org/0000-0002-8273-2146) ${ }^{5}$
}

\begin{abstract}
Resumo
Imigrantes e refugiados são uma população de risco para transtornos mentais, como o Transtorno de Estresse PósTraumático (Tept), e a psicoterapia com indivíduos provenientes de diferentes contextos culturais pode ser desafiadora, porém, a Terapia Cognitivo-Comportamental (TCC) vem se mostrando uma abordagem eficaz com essa população. O presente estudo de revisão sistemática tem como objetivo descrever os protocolos culturalmente adaptados de TCC para imigrantes e refugiados com diagnóstico ou sintomas de Tept, seguindo as recomendações do método PRISMA. Foram realizadas buscas nas bases de dados PubMed/Medline e PsycInfo, nos últimos 15 anos, sendo incluídos 11 artigos. As principais adaptações realizadas pelos estudos foram: o uso da língua mãe dos participantes, uso de imagens relativas ao país de origem ou à religião e modificações na exposição usual. As informações descritas neste estudo podem auxiliar no desenvolvimento de futuros estudos ou na prática clínica com essa população.
\end{abstract}

Palavras-chave: Transtorno de Estresse Pós-Traumático. Terapia Cognitivo-Comportamental. Imigrantes. Refugiados.

\footnotetext{
1 Pontifícia Universidade Católica do Rio Grande do Sul, Porto Alegre, Brasil. E-mail: brunnetalice@gmail.com.

2 Pontifícia Universidade Católica do Rio Grande do Sul, Porto Alegre, Brasil. E-mail: laura.bolasell@acad.pucrs.br.

3 Pontifícia Universidade Católica do Rio Grande do Sul, Porto Alegre, Brasil. E-mail: marcelobujak@gmail.com.

4 Pontifícia Universidade Católica do Rio Grande do Sul, Porto Alegre, Brasil. E-mail: marcelo.m.rigoli@gmail.com.

5 Pontifícia Universidade Católica do Rio Grande do Sul, Porto Alegre, Brasil. E-mail: christian.kristensen@pucrs.br.
} 


\begin{abstract}
Immigrants and refugees are a population at risk for mental disorders, such as Posttraumatic Stress Disorder (PTSD), and psychotherapy with individuals from different cultural contexts can be challenging, however, Cognitive-Behavioral Therapy (CBT) has shown to be an effective approach with this population. This systematic review study aims to describe the culturally adapted protocols of CBT for immigrants and refugees with diagnosis or symptoms of PTSD, following the recommendations of the PRISMA method. Searches were conducted in the PubMed/Medline and PsycInfo databases, in the last 15 years, and 11 articles were included. The main adjustments made by the studies were: the use of the participant's mother tongue, use of images related to the country of origin or religion and modifications in the usual exposure. The information described in this study may help in the development of future studies or in clinical practice with this population.
\end{abstract}

Keywords: Posttraumatic Stress Disorder. Cognitive-Behavioral Therapy. Immigrants. Refugees.

O mundo está vivenciando um fluxo migratório internacional sem precedentes, fazendo com que milhares de pessoas passem a viver nas grandes cidades (International Organization for Migration, 2015). Esses indivíduos fogem da violência de seus países de origem, mas alguns acabam encontrando dificuldades também nos países de destino, como preconceito, pobreza e abuso. Os eventos potencialmente traumáticos vivenciados no país de origem, somados com as dificuldades encontradas após a migração, colocam essa população em risco para o desenvolvimento de Transtorno de Estresse Pós-Traumático (Abebe, Lien \& Hjelde, 2012; Fazel, Wheeler \& Danesh, 2005). Tendo em vista os problemas de saúde mental, diversos estudos, principalmente com refugiados, já foram conduzidos com o objetivo de verificar o efeito da Terapia Cognitivo-Comportamental (TCC) em minorias étnicas e com resultados promissores (Crumlish \& O’Rourke, 2010; Gwozdziewycz \& Mehl-Madrona, 2013; Lambert \& Alhassoon, 2015; Palic \& Elklit, 2011). No entanto, ainda são necessários estudos sobre os fatores culturais e suas influências na psicoterapia com essa população (Huey, Tilley, Jones \& Smith, 2014; Lambert \& Alhassoon, 2015).

Em uma metanálise, encontrou-se um efeito médio da Terapia de Exposição Narrativa (NET) para refugiados. Estudos que utilizaram refugiados treinados como terapeutas apresentaram maior tamanho de efeito do que aqueles em que os terapeutas foram psicólogos de outras nacionalidades (Gwozdziewycz \& Mehl-Madrona, 2013). No entanto, a metanálise contém apenas sete artigos, e apenas três em adultos, sendo todos 
dos mesmos autores (Neuner et al., 2010, 2008; Neuner, Schauer, Klaschik, Karunakara \& Elbert, 2004). Outra revisão sistemática que incluiu apenas ensaios clínicos controlados e randomizados $(n=10)$ encontrou estudos com populações diversas, mas que apresentavam, em sua maioria, protocolos de TCC ou NET. Os autores concluíram que os estudos analisados não apresentam evidências sólidas da eficácia dos tratamentos (Crumlish \& O’Rourke, 2010). Por outro lado, a TCC mostrou bons resultados em uma revisão sistemática realizada por Palic \& Elklit (2011), que incluiu 25 estudos e investigou o efeito de tratamentos psicossociais para Tept em refugiados. Os resultados foram promissores, principalmente no que diz respeito a dois subtipos de tratamentos: a NET (Neuner et al., 2008) e a TCC culturalmente adaptada (Hinton et al., 2004, 2005, 2009).

Outro estudo de metanálise investigou os resultados de estudos randomizados e controlados de intervenções focadas no trauma para refugiados e os possíveis fatores associados ao tamanho de efeito da intervenção nos sintomas de Tept. Foram utilizados protocolos de NET, TCC e EMDR nos estudos incluídos e eles apresentaram grandes tamanhos de efeito para a redução de Tept. O uso de tradutores e os instrumentos utilizados para mensurar os sintomas de Tept não apresentaram efeitos no desfecho dos tratamentos. Por outro lado, o tamanho de efeito da intervenção foi maior nos estudos com maior número de sessões e quando o grupo de comparação era uma condição passiva (por exemplo: lista de espera). Os autores ressaltam que são necessários mais estudos sobre os fatores que podem interferir no desfecho da psicoterapia, como as questões culturais (Lambert \& Alhassoon, 2015).

Para o estudo das questões culturais, deve-se delimitar o conceito de cultura, definida neste estudo como um conjunto dinâmico de crenças, valores e comportamentos compartilhados entre indivíduos que dividem os mesmos espaços. No entanto, diversos países estão vivendo em ambientes chamados "multiculturais", principalmente em razão dos fluxos migratórios. Nesses ambientes, a cultura deixa de ser algo "invisível", pois indivíduos que agora compartilham os mesmos locais já construíram crenças e valores nos seus países de origem. Elementos da cultura têm um grande impacto na saúde mental dos indivíduos, desde a noção de saúde mental até o gerenciamento desta: tomar medicação, ir ao médico, 
tentar lidar sozinho com o problema, etc. (Alegria, Atkins, Farmer, Slaton \& Stelk, 2010). O desenvolvimento de protocolos culturalmente adaptados, portanto, podem favorecer um melhor desfecho na atenção psicossocial a imigrantes e refugiados.

O modelo de adaptação cultural utilizado no presente estudo é o de Bernal e colaboradores (Bernal, Bonilla \& Bellido, 1995; Bernal, Jiménez-Chafey \& Domenech Rodríguez, 2009). Segundo os autores, a adaptação cultural de protocolos de psicoterapia consiste em modificações sistemáticas em um tratamento baseado em evidências para considerar questões como crenças, valores e significados específicos de uma determinada população. Para tanto, eles sugerem alterações nos seguintes domínios: linguagem, pessoas (terapeuta ter a mesma origem étnica do paciente), metáforas adequadas à cultura, aquisição de conhecimento cultural, apresentação dos problemas congruentes com o sistema de crenças do paciente, objetivos acordados entre terapeuta e paciente, compatibilidade entre os métodos de tratamento e a cultura do paciente e, por fim, consideração com o contexto em que o paciente está atualmente inserido (Bernal et al., 1995; Huey et al., 2014).

A partir da revisão anterior da literatura, o presente estudo tem como objetivo descrever as adaptações culturais de protocolos de intervenção para o Transtorno de Estresse Pós-Traumático baseados em Terapia Cognitivo-Comportamental. Para tanto, será realizada uma revisão sistemática de estudos que descrevem as técnicas utilizadas e suas adaptações. Objetiva-se, também, discutir a forma de avaliação da sintomatologia nessa população, bem como as vantagens e limitações das técnicas descritas, a fim de contribuir para o desenvolvimento de futuros estudos e no avanço da prática clínica.

\section{Método}

A presente revisão sistemática foi realizada a partir das recomendações do PRISMA (Mother, Liberati, Tetzlaff \& Altman, 2009). Foram realizadas buscas nas bases de dados PubMed/Medline e Psyclnfo, por meio da seguinte estratégia de busca (psychotherapy OR counselling OR cognitive behaviour therapy) AND (transcultural OR crosscultural OR refugee OR refugees OR migrants OR migrant OR immigrant OR immigrants) AND (posttraumatic 
stress disorder OR PTSD). Foram incluídos artigos empíricos que (i) relatavam protocolos baseados em Terapia Cognitivo-Comportamental (TCC) para o Transtorno de Estresse PósTraumático culturalmente adaptados para imigrantes ou refugiados (ii) em população adulta, (iii) publicados nos últimos 15 anos, (iv) artigos em língua inglesa, espanhola, francesa ou italiana.

A avaliação dos critérios de inclusão foi realizada em duas etapas. Inicialmente, os resumos foram lidos e avaliados de forma independente por dois juízes, autores do presente estudo, com experiência em Terapia Cognitivo-Comportamental (TCC), saúde mental e imigração. Após essa etapa, os autores chegaram a um consenso sobre a inclusão ou não dos resumos com discordância na etapa anterior.

\section{Resultados}

\section{Descrição geral dos estudos}

A Figura 1 descreve o processo de busca e seleção dos artigos. Os artigos incluídos são empíricos e descrevem adaptações culturais de protocolos de psicoterapia para imigrantes e/ou refugiados. Cabe ressaltar que os estudos que não descreveram as técnicas utilizadas ou não explicitaram a intenção da realização de uma adaptação cultural do protocolo apresentado foram excluídos. Por isso, foram incluídos 11 artigos, em língua inglesa. Os critérios utilizados visaram à inclusão de estudos que contavam com métodos rigorosos, no entanto, não foi realizada uma avaliação de risco de viés nos ensaios clínicos incluídos na presente revisão.

$\mathrm{Na}$ amostra final, cinco estudos são oriundos de um mesmo grupo de pesquisa, mas têm amostras diferentes (Hinton et al., 2004, 2005; Hinton, Hofmann, Rivera, Otto \& Pollack, 2011 ; Hinton, Ojserkis, Jalal, Peou \& Hofmann, 2013; Hinton, Pich, Chhean, Safren \& Pollack, 2006). Estudos que utilizavam Eye Moviment Desintization and Reprocessing (EMDR) também foram incluídos, pois esse protocolo também é realizado a partir de técnicas em comum com a TCC. 
Figura 1. Processo de busca e seleção de artigos

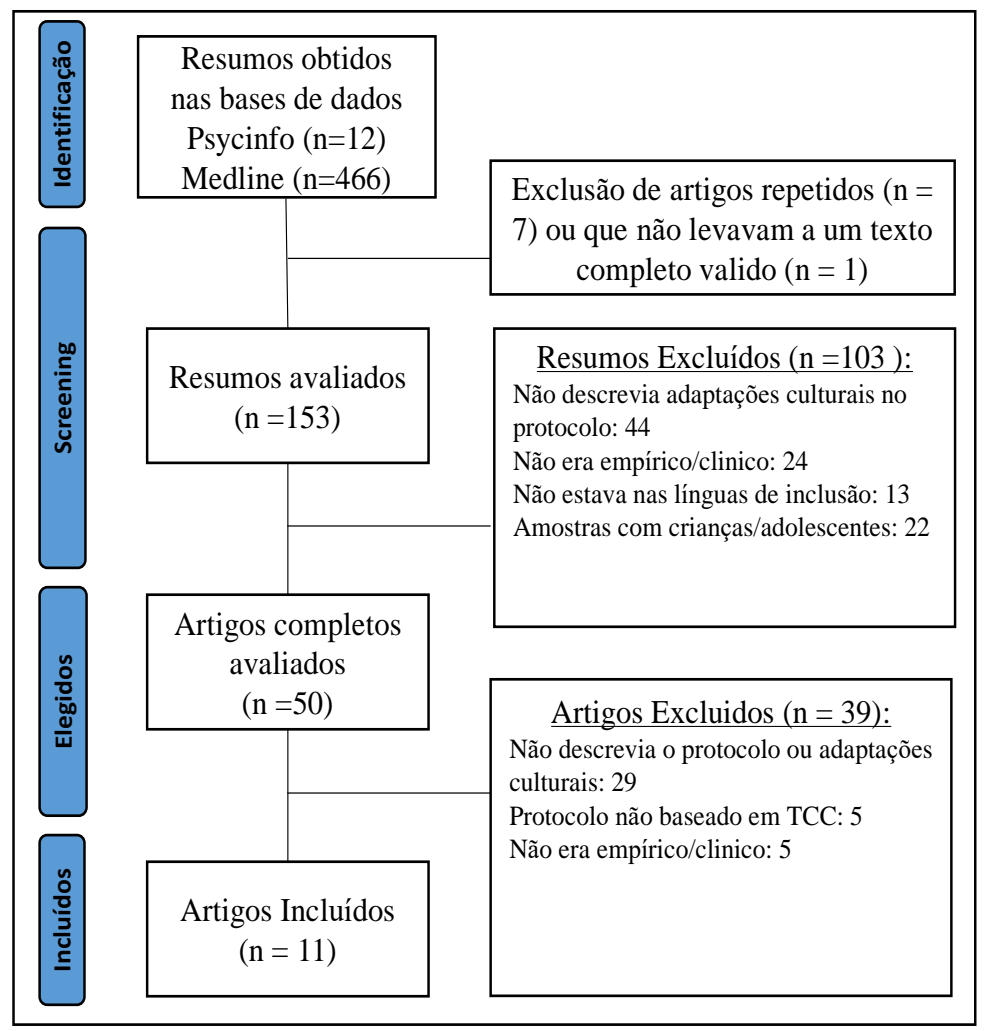

Fonte: Elaborada pelos autores.

A Tabela 1 descreve os estudos incluídos. Os estudos relataram protocolos baseados em TCC focada no trauma, EMDR, Terapia de Exposição Narrativa (NET), Mindfulness e Terapia de Aceitação e Compromisso (ACT). A avaliação da sintomatologia de Tept foi realizada pela maioria dos estudos por meio de escalas de autorrelato, principalmente o Harvard Trauma Questionnaire (HTQ; Mollica et al., 1992).Todos os estudos descreveram que o processo terapêutico foi realizado na língua mãe dos participantes. Em quatro estudos, foi feito o uso de intérpretes (Acarturk et al., 2015; Buhmann et al., 2015; Drožđek, Kamperman, Tol, Knipscheer \& Kleber, 2014; Schouler-Ocak, Reiske, Rapp \& Heinz, 2008). Nos estudos de Hinton e colaboradores (2005, 2006, 2011 e 2013) e Kruse e colaboradores (2009) os tratamentos foram conduzidos por psicoterapeutas fluentes na língua dos participantes. No estudo de Neuner e colaboradores (2008), nove refugiados foram treinados como aconselhadores para conduzirem o protocolo de tratamento na língua 
mãe dos participantes. De uma forma geral, os protocolos descritos continham os elementos básicos da TCC focada no trauma (Foa \& Rothbaum, 2001): psicoeducação, relaxamento, exposição e técnicas para reestruturação cognitiva. As técnicas de psicoeducação consistiam em explicações para o paciente sobre o Tept, imigração, o tratamento a ser oferecido, entre outros. Tais técnicas não foram mencionadas em apenas um artigo (Hinton et al., 2013). 
Tabela 1. Estudos incluídos

\begin{tabular}{|c|c|c|c|c|c|c|c|}
\hline & Estudo & País do estudo & País da amostra & Modalidade & Design & $\mathbf{N}$ & Escalas \\
\hline 1 & Buhmann et al., 2015 & Dinamarca & $\begin{array}{l}\text { Iraque, Irã, Líbano, Palestina e } \\
\text { outros }\end{array}$ & Individual & ECA & 85 & HTQ \\
\hline 2 & Acarturk et al., 2015 & Turquia & Síria & Individual & ECC & $\begin{array}{l}\mathrm{GE}=15 \\
\mathrm{GC}=14\end{array}$ & IES-R \\
\hline 3 & Drožđek et al., 2014 & Holanda & Irã e Afeganistão & Grupo & ECA & 69 & HTQ \\
\hline 4 & Hinton et al., 2013 & EUA & Camboja & Individual & EC & 1 & - \\
\hline \multirow[t]{2}{*}{5} & Hinton et al., 2011 & EUA & República Dominicana e Porto Rico & Individual & ECCR & $\mathrm{GE}=12$ & PTSD \\
\hline & & & & & & $\mathrm{GC}=12$ & Checklist \\
\hline \multirow[t]{2}{*}{6} & Kruse, et al., 2009 & Alemanha & Bósnia & Individual & ECC & $\mathrm{GE}=35$ & HTQ \\
\hline & & & & & & $G C=35$ & \\
\hline 7 & Schouler-Ocak et al., 2008 & Alemanha & Turquia & Individual & SEC & 2 & CID-10 \\
\hline 8 & Neuner et al., 2008 & Uganda & Ruanda e Somália & Individual & ECCR & $G E=111$ & PDS \\
\hline
\end{tabular}


111

GC $2=55$

\begin{tabular}{|c|c|c|c|c|c|c|c|}
\hline 9 & Hinton et al., 2006 & EUA & Camboja & Individual & SEC & 3 & HTQ \\
\hline \multirow[t]{2}{*}{10} & Hinton et al., 2005 & EUA & Camboja & Individual & ECCR & $\mathrm{GE}=20$ & CAPS \\
\hline & & & & & & $\mathrm{GC}=20$ & \\
\hline \multirow[t]{2}{*}{11} & Hinton et al., 2004 & EUA & Vietnã & Individual & ECCR & $\mathrm{GE} 1=6$ & HTQ \\
\hline & & & & & & GE2-6 & \\
\hline
\end{tabular}

Nota: ECA = Ensaio Clinico Aberto; ECC= Ensaio Clínico Controlado; ECCR = Ensaio Clínico Controlado e Randomizado; EC = Estudo de Caso; SEC = Série de estudos de casos. GE = Grupo Experimental; GC = Grupo Controle; HTQ = Harvard Trauma Questionnaire; IES-R = Impact of Events ScaleRevised; PDS = The Post Traumatic Stress Diagnostic Scale; CAPS = Clinician-Administred PTSD Scale.

Fonte:

Elaborada

pelos

autores. 


\section{Descrição das técnicas utilizadas}

O uso de técnicas de relaxamento e de Mindfulness só não foi citado por um estudo (Neuner et al., 2008). Sete estudos fizeram uso de princípios de Mindfulness (Buhmann et al., 2015; Hinton et al., 2004, 2005, 2011 ; Hinton et al., 2013; Hinton et al., 2006). Os outros estudos relataram uso de respiração diafragmática (Hinton et al., 2004, 2005, 2011 ; Hinton et al., 2013; Hinton et al., 2006), relaxamento por imagens (Acarturk et al., 2015; Hinton et al., 2011, 2006), relaxamento muscular (Hinton et al., 2004, 2011; Hinton et al., 2013; Kruse et al., 2009). Dois estudos não especificaram as técnicas de relaxamento utilizadas (Buhmann et al., 2015; Drožđek, Kamperman, Tol, Knipscheer \& Kleber, 2014). Foram usada técnicas de relaxamento utilizando imagens de paisagens do país de origem dos participantes ou vinculadas à religião destes (Hinton et al., 2004, 2005, 2006, 2011, 2013; Arcaturk et al., 2015; Kruse et al., 2009). As técnicas de exposição utilizadas variaram entre os estudos, sendo que um estudo não relatou o uso de exposição (Kruse et al., 2009). A exposição imagística (Foa \& Rothbaum, 2001), na qual o paciente relata a situação traumática vivenciada, foi utilizada em dois estudos (Buhmann et al., 2015; Drožđek et al., 2014). Quatro estudos de Hinton e colaboradores (2005, 2011, 2013, 2006) descrevem o uso de exposição imagística e narrativa (Brewin, Dalgleish \& Joseph, 1996; Clark \& Ehlers, 2004; Foa \& Rothbaum, 2001), mas usualmente acompanhados por técnicas de regulação emocional, tais como: aceitação, meditação baseada em Mindfulness e técnicas de relaxamento. Já em seu outro estudo, Hinton et al. (2004), faz uso de Exposição Interoceptiva, em que os participantes foram expostos a suas sensações corporais associadas aos ataques de pânico gerados pelas associações traumáticas (Falsetti \& Resnick, 2000; Otto, Penava, Pollack \& Smoller, 1996). Este estudo ainda utiliza o protocolo de regulação emocional (Foa \& Rothbaum, 2001; Rachman, 1980) para trabalhar revivências e flashbacks e estimular a mudança de uma visão dolorosa para uma consciência multisensorial do momento presente (mindfulness). Schouler-Ocak e colaboradores (2008) descrevem o uso de uma técnica chamada "television-screen method" (Reddemann \& Sachsse, 1997), em que o paciente imagina que a história traumática está acontecendo em uma televisão, da qual ele possui o controle remoto e pode modificar o canal ou a velocidade com que as imagens são passadas. O paciente também é incentivado a se lembrar dos pensamentos, sentimentos e sensações corporais associadas ao "filme". Por fim, Neuner e 
colaboradores (2008) utilizaram a exposição narrativa, a qual é uma adaptação da terapia de exposição tradicional, para os indivíduos vítimas de guerras e tortura. É realizada uma narrativa da biografia do indivíduo, incluindo os diversos eventos traumáticos vivenciados.

Técnicas de reestruturação cognitiva foram utilizadas em todos os estudos. Os autores relatam o uso de técnicas como questionamento de crenças, registro e modificação de pensamentos distorcidos, entre outros. No protocolo de EMDR, conduzido por Acarturk e colaboradores (2015), a reestruturação cognitiva foi feita por meio da instalação de uma crença positiva, também com a prática de movimentos oculares bilaterais. Na NET, a reestruturação cognitiva é realizada juntamente com o relato biográfico do indivíduo (Neuner et al., 2008). Nos estudos de Hinton e colaboradores (2005, 2006, 2011), a reestruturação cognitiva tinha um grande foco nas sensações físicas de ansiedade e nas síndromes culturais (ataque de nervios), problema frequente na população de seus estudos. Da mesma forma, Schouler-Ocak e colaboradores (2008) e Kruse e colaboradores (2009) descrevem técnicas de reestruturação cognitiva e resolução de problemas compatíveis com a cultura do participante, por exemplo, a crença existente em algumas culturas de que mulheres que sofreram uma situação de violência sexual trazem desgraça à família (Kruse et al., 2009). Schouler-Ocak e colaboradores (2008) destacam também que o contato com uma nova cultura pode gerar questionamento de alguns valores pessoais.

\section{Resultados de eficácia}

Entre os estudos incluídos, quatro foram ensaios clínicos controlados e randomizados. No estudo realizado por Hinton e colaboradores (2011), mulheres latinas com Tept resistente a tratamento foram submetidas a um protocolo de TCC adaptada culturalmente. As participantes foram alocadas de forma randomizada no grupo de TCC ou de relaxamento muscular aplicado (AMR). Ambas as condições foram eficazes para redução da sintomatologia de Tept, no entanto, o grupo TCC apresentou escores significativamente menores no pós-tratamento $(d=1,6)$. A TCC adaptada ao trauma também se mostrou efetiva para refugiados do Camboja diagnosticados com Tept e Transtorno de Pânico, na comparação com lista de espera, mesmo no follow-up $(d=2,17$; Hinton et al., 2005). No estudo de Hinton e colaboradores (2004), os participantes vietnamitas 
foram divididos em dois grupos de seis: tratamento imediato (Grupo 1) e tratamento tardio (Grupo 2) no mesmo protocolo de TCC adaptada culturalmente. Na comparação dos grupos, o Grupo 1 apresentou uma maior redução nos sintomas de Tept, ansiedade e pânico do que o Grupo 2 ( $d=$ 2,5), porém este também apresentou reduções significativas nos sintomas. Neuner e colaboradores (2008) compararam o efeito de três condições: NET, aconselhamento para trauma (TC) e um grupo controle, sem intervenção. Ambos os tratamentos ativos foram mais eficazes do que o grupo controle $(d=0.8)$, no follow-up de seis meses, no entanto, não houve diferença entre a NET $(d=1,4)$ e o TC $(d=1,5)$.

Dois estudos relataram ensaios clínicos controlados (Acarturk et al., 2015; Kruse et al., 2009). No estudo que comparou o tratamento com EMDR e lista de espera para refugiados Sírios, o grupo experimental apresentou significativamente menores índices de sintomas de Tept no póstratamento ( $d=1,78$; Acartuk et al., 2015). Kruse e colaboradores (2009) testaram o efeito da TCC focada no trauma para refugiados da Bósnia, comparando-a com o tratamento usual. A TCC apresentou um maior tamanho de efeito quando comparado com o tratamento usual (respectivamente, Cohen's $d=2,7$ e Cohen's $d=-0,3$ ).

Dois estudos apresentaram ensaios clínicos abertos (Buhmann et al., 2015; Drožđek et al., 2014). No estudo de Buhmann e colaboradores (2015), o qual avaliou o efeito da TCC para Tept em refugiados de diversos países, não foram encontradas diferenças significativas entre o pré e o pós-tratamento nos sintomas de Tept. Drožđek e colaboradores (2014) investigaram o efeito da TCC focada no trauma em grupo. A sintomatologia de Tept foi signitivativamente mais baixa em follow-up de sete anos em relação ao pré-tratamento $(p<0,01)$.

Três estudos apresentaram resultados de estudos de caso ou série de estudos de casos (Hinton, Ojserkis, et al., 2013; Hinton et al., 2006; Schouler-Ocak et al., 2008). Entre eles, dois apresentam apenas resultados qualitativos. No primeiro (Hinton et al., 2013), foi apresentado um caso para ilustrar a aplicação da terapia baseada em Loving-kindness. No segundo estudo, Schouler-Ocak e colaboradores (2008) descrevem, a partir de dois casos, as especificidades culturais do tratamento com TCC focada no trauma e EMDR para imigrantes. O estudo de séries de casos realizado por Hinton e colaboradores (2006) apresentou três casos de participantes submetidos ao tratamento com foco somático em refugiados traumatizados do Camboja, com 
diagnóstico de Tept e ataques de pânico. Os participantes apresentaram ao menos 36\% de melhora nos sintomas de Tept.

\section{Discussão}

O presente estudo objetivou descrever as adaptações culturais dos protocolos baseados em TCC para Tept em imigrantes e refugiados. As principais adaptações realizadas pelos estudos foram as seguintes: o uso da língua mãe dos participantes, uso de imagens relativas ao país de origem ou à religião e modificações na exposição usual. Os protocolos testados por meio de ensaios clínicos controlados mostraram bons resultados (Acarturk et al., 2015; Hinton et al., 2004, 2005, 2011; Kruse et al., 2009; Neuner et al., 2008). Entre os ensaios clínicos abertos, um dos estudos não relatou efetividade do protocolo utilizado para os sintomas de Tept (Buhmann et al., 2015).

O rastreio da sintomatologia de Tept foi realizada, na maior parte dos estudos, por escalas de autorrelato. Schouler-Ocak e colaboradores (2008) relataram dificuldades em realizar o diagnóstico de Tept de acordo com os critérios da CID-10. Os autores enfatizam também que o uso de escalas para o diagnóstico do transtorno pode estar equivocado, visto que a maioria dos instrumentos é realizada a partir de uma ideia ocidental de psicopatologia. A questão da aplicabilidade do diagnóstico de Tept proposto pelo DSM-5 e pela CID-10 ainda é controversa, tendo duas vertentes de pensamento: uma descreve o Tept como um diagnóstico influenciado pela cultura ocidental e que não seria aplicado para pacientes não ocidentais; a outra vertente diz que o Tept é um diagnóstico que pode ser aplicado nos diferentes contextos culturais. Encontra-se também na literatura ideias que ponderam essa questão do diagnóstico, assumindo que existem respostas traumáticas universais, mas que a vulnerabilidade, a expressão e a resposta ao tratamento do Tept podem variar entre as culturas (Kienzler, 2008; Palic \& Elklit, 2011; Tay, Rees, Chen, Kareth \& Silove, 2015). Nesse sentido, Hinton e colaboradores (2013; 2011) propõem que os critérios expostos pelo DSM incluem uma parte fundamental da resposta universal ao trauma; no entanto, algumas especificidades de expressão do transtorno ou de comorbidades, como as síndromes culturais (ataque de nervios), não estavam incluídas nas versões anteriores do DSM. Atualmente, o DSM-5 dispõe de ferramentas como a Entrevista de Formulação Cultural, as quais 
visam aprimorar a sensibilidade cultural nos diagnósticos de saúde mental (American Psychiatric Association, 2013).

Todos os estudos da presente revisão fizeram ou uso de intérpretes ou terapeutas com o domínio da língua mãe dos participantes. Considerando a dificuldade de que os terapeutas tenham o domínio da língua e conhecimentos específicos de uma cultura diferente da sua, o uso de intérpretes pode ser essencial para o andamento do tratamento (Bernal et al., 2009; WenkAnsohn \& Gurris, 2011 1). Além disso, Lambert e colaboradores (2015) não encontraram diferenças significativas nos resultados de estudos que foram conduzidos apenas pelos terapeutas na língua dos participantes e aqueles que fizeram uso de tradutores.

Apesar das evidências de eficácia da terapia de exposição para o Tept, alguns estudos incluídos na presente revisão reportam dificuldades no uso da técnica com alguns grupos de imigrantes ou refugiados. Buhmann e colaboradores (2015) relataram que a exposição foi utilizada com menos frequência pelos terapeutas do que o planejado no manual, pois os pacientes se negaram devido aos seus altos níveis de angústia e, nas sessões traduzidas, houve pouco tempo para uma exposição visual adequada. A exposição prolongada pode não ser tolerada por alguns grupos étnicos, gerando grandes níveis de drop-out (Hinton, Rivera, Hofmann, Barlow \& Otto, 2012). Por isso, os protocolos propostos por Hinton e colaboradores envolvem também estratégias de regulação emocional, como técnicas de relaxamento e também técnicas baseadas em Mindfulness. Buhmann e colaboradores (2015), por outro lado, encontraram uma mudança negativa na qualidade de vida e no nível de funcionalidade dos pacientes associada ao uso de técnicas de relaxamento baseadas em Mindfulness, questionando o efeito do uso dessas técnicas em terapia. No entanto, os autores relatam também que o uso dessas técnicas foi realizado com aqueles pacientes que apresentavam maior sofrimento, o que pode ter influenciado os resultados. Nos outros estudos, não foram relatados efeitos adversos do uso de técnicas de relaxamento.

O presente estudo apresentou as técnicas utilizadas com imigrantes em protocolos ditos como culturalmente sensíveis. Algumas limitações podem ser encontradas, como a inclusão apenas de artigos que citavam alguma adaptação cultural e que descreviam detalhadamente o protocolo, pois, dessa forma, alguns estudos com adaptações importantes podem ter sido excluídos. Acredita-se, no entanto, que as informações descritas neste estudo podem auxiliar no desenvolvimento de futuros estudos ou na prática clínica com essa população. 


\section{Referências}

Abebe, D. S., Lien, L., \& Hjelde, K. H. (2012). What We Know and Don't Know About Mental Health Problems among Immigrants in Norway. Journal of Immigrant and Minority Health, 16, 6067. https://doi.org/10.1007/s10903-012-9745-9.

Acarturk, C., Konuk, E., Cetinkaya, M., Senay, I., Sijbrandij, M., Cuijpers, P., \& Aker, T. (2015). EMDR for Syrian Refugees with Posttraumatic Stress Disorder Symptoms: Results of a Pilot Randomized Controlled Trial. European Journal of Psychotraumatology, 6, Article 27414. https://doi.org/10.3402/ejpt.v6.27414.

Alegria, M., Atkins, M., Farmer, E., Slaton, E., \& Stelk, W. (2010). One Size does not Fit All: Taking Diversity, Culture and Context Seriously. Administration and Policy in Mental Health and Mental Health Services Research, 37(1-2), 48-60. https://doi.org/10.1007/s10488-0100283-2.

American Psychiatric Association. (2013). Diagnostic and Statistical Manual of Mental Disorders (5th ed.). Washington, DC: American Psychiatric Association.

Bernal, G., Bonilla, J., \& Bellido, C. (1995). Ecological Validity and Cultural Sensitivity for Outcome Research - Issues for the Cultural-Adaptation and Development of Psychosocial Treatments with Hispanics. Journal of Abnormal Child Psychology, 23(1), 67-82. https://doi.org/10.1017/СВО9781107415324.004.

Bernal, G., Jiménez-Chafey, M. I., \& Domenech Rodríguez, M. M. (2009). Cultural Adaptation of Treatments: A Resource for Considering Culture in Evidence-Based Practice. Professional Psychology: Research and Practice, 40(4), 361-368. https://doi.org/10.1037/a0016401.

Brewin, C., Dalgleish, T., \& Joseph, S. (1996). A Dual Representation Theory of Posttraumatic Stress Disorder. Psychological Review, 670-686. https://doi.org/10.1037//0033295X.103.4.670.

Buhmann, C., Andersen, I., Mortensen, E. L., Ryberg, J., Nordentoft, M., \& Ekstrøm, M. (2015). Cognitive Behavioral Psychotherapeutic Treatment at a Psychiatric Trauma Clinic for 
Refugees: Description and Evaluation. Torture, 25(1), 17-32. Retrieved from http://www.ncbi.nlm.nih.gov/PubMed/26021345.

Clark, D. M., \& Ehlers, A. (2004). Posttraumatic Stress Disorder: From Theory to Practice. In R. H. Leahy (Ed.). Contemporary Cognitive Therapy: Theory Research and Practice.

Crumlish, N., \& O'Rourke, K. (2010). A Systematic Review of Treatments for Post-Traumatic Stress Disorder among Refugees and Asylum-Seekers. The Journal of Nervous and Mental Disease, 198(4), 237-251. https://doi.org/10.1097/NMD.0b013e3181d61258.

Drožđek, B., Kamperman, A. M., Tol, W. a., Knipscheer, J. W., \& Kleber, R. J. (2014). Seven-Year Follow-Up Study of Symptoms in Asylum Seekers and Refugees With PTSD Treated With Trauma-Focused Groups. Journal of Clinical Psychology, 70(4), 376-387. https://doi.org/10.1002/jclp.22035.

Fazel, M., Wheeler, J., \& Danesh, J. (2005). Prevalence of Serious Mental Disorder in 7000 Refugees Resettled in Western Countries: A Systematic Review. Lancet, 365(panel 1), 1309-1314. https://doi.org/10.1016/S0140-6736(05)61027-6.

Foa, E. B., \& Rothbaum, B. O. (2001). Treating the Trauma of Rape: Cognitive-Behavioral Therapy for PTSD. Guilford Press.

Gwozdziewycz, N., \& Mehl-Madrona, L. (2013). Meta-Analysis of the Use of Narrative Exposure Therapy for the Effects of Trauma among Refugee Populations. The Permanente Journal, l7(1), 70-76. https://doi.org/10.7812/TPP/12-058.

Hinton, D. E., Chhean, D., Pich, V., Safren, S. a., Hofmann, S. G., \& Pollack, M. H. (2005). A Randomized Controlled Trial of Cognitive-Behavior Therapy for Cambodian Refugees with Treatment-Resistant PTSD and Panic Attacks: A Cross-Over Design. Journal of Traumatic Stress, 18(6), 617-629. https://doi.org/10.1002/jts.20070.

Hinton, D. E., Hofmann, S. G., Rivera, E., Otto, M. W., \& Pollack, M. H. (2011). Culturally Adapted CBT (CA-CBT) for Latino Women with Treatment-Resistant PTSD: A Pilot Study Comparing CA-CBT to Applied Muscle Relaxation. Behaviour Research and Therapy, 49(4), 275-280. 
https://doi.org/10.1016/j.brat.2011.01.005.

Hinton, D. E., Kredlow, M. A., Pich, V., Bui, E., \& Hofmann, S. G. (2013). The Relationship of PTSD to Key Somatic Complaints and Cultural Syndromes among Cambodian Refugees Attending a Psychiatric Clinic: The Cambodian Somatic Symptom and Syndrome Inventory (CSSI). Transcultural Psychiatry, 50(3), 347-370. https://doi.org/10.1177/1363461513481187.

Hinton, D. E., \& Lewis-Fernández, R. (2011). The Cross-Cultural Validity of Posttraumatic Stress Disorder: Implications for DSM-5. Depression and Anxiety, 28(9), 783-801. https://doi.org/10.1002/da.20753.

Hinton, D. E., Ojserkis, R. a., Jalal, B., Peou, S., \& Hofmann, S. G. (2013). Loving-Kindness in the Treatment of Traumatized Refugees and Minority Groups: A Typology of Mindfulness and the Nodal Network Model of Affect and Affect Regulation. Journal of Clinical Psychology, 69(8), 817-828. https://doi.org/10.1002/jclp.22017.

Hinton, D. E., Pich, V., Chhean, D., Safren, S. a., \& Pollack, M. H. (2006). Somatic-Focused Therapy for Traumatized Refugees: Treating Posttraumatic Stress Disorder and Comorbid NeckFocused Panic Attacks among Cambodian Refugees. Psychotherapy: Theory, Research, Practice, Training, 43(4), 491-505. https://doi.org/10.1037/0033-3204.43.4.491.

Hinton, D. E., Rivera, E. I., Hofmann, S. G., Barlow, D. H., \& Otto, M. W. (2012). Adapting CBT for Traumatized Refugees and Ethnic Minority Patients: Examples from Culturally Adapted CBT (CA-CBT). Transcultural Psychiatry, 49(2), 340-365. https://doi.org/10.1177/1363461512441595.

Huey, S. J., Tilley, J. L., Jones, E. O., \& Smith, C. A. (2014). The Contribution of Cultural Competence to Evidence-Based Care for Ethnically Diverse Populations. Annu. Rev. Clin. Psychol, 10, 305-338. https://doi.org/10.1146/annurev-clinpsy-032813-153729.

International Organization for Migration, I. (2015). Migrants and Cities: New Partnerships to Manage Mobility. Geneva.

Kienzler, H. (2008). Debating War-Trauma and Post-Traumatic Stress Disorder (PTSD) in an 
Interdisciplinary Arena. Social Science \& Medicine, 67(2), 218-227.

https://doi.org/10.1016/j.socscimed.2008.03.030.

Kruse, J., Joksimovic, L., Cavka, M., Woller, W., \& Schmitz, N. (2009). Effects of Trauma-Focused Psychotherapy upon War Refugees. Journal of Traumatic Stress, 23(2), 585-592. https://doi.org/10.1002/jts.20477

Lambert, J. E., \& Alhassoon, O. M. (2015). Trauma-Focused Therapy for Refugees: Meta-Analytic Findings. Journal of Counseling Psychology, 62(1), 28-37. https://doi.org/10.1037/cou0000048.

Mollica, R. F., Caspi-Yavin, Y., Bollini, P., Truong, T., Tor, S., \& Lavelle, J. (1992). The Harvard Trauma Questionnaire: Validating a Cross-Cultural Instrument for Measuring Torture, Trauma, and Posttraumatic Stress Disorder in Indochinese Refugees. The Journal of Nervous and Mental Disease, 180(2), 111-116.

Mother, D., Liberati, A., Tetzlaff, J., \& Altman, D. (2009). Preferred Reporting Items for Systematic Reviews and Meta-Analyses: The PRISMA Statement. Retrieved March 31, 2015, from Annals of Internal Medicine. Retrieved from file:///C:/Users/Alice/Downloads/0000605200908180-00008.pdf.

Neuner, F., Kurreck, S., Ruf, M., Odenwald, M., Elbert, T., \& Schauer, M. (2010). Can AsylumSeekers with Posttraumatic Stress Disorder be Successfully Treated? A Randomized Controlled Pilot Study. Cognitive Behaviour Therapy, 39(2), 81-91. https://doi.org/10.1080/16506070903121042.

Neuner, F., Onyut, P. L., Ertl, V., Odenwald, M., Schauer, E., \& Elbert, T. (2008). Treatment of Posttraumatic Stress Disorder by Trained Lay Counselors in an African Refugee Settlement: A Randomized Controlled Trial. Journal of Consulting and Clinical Psychology, 76(4), 686694. https://doi.org/10.1037/0022-006X.76.4.686.

Neuner, F., Schauer, M., Klaschik, C., Karunakara, U., \& Elbert, T. (2004). A Comparison of Narrative Exposure Therapy, Supportive Counseling, and Psychoeducation for Treating Posttraumatic Stress Disorder in an African Refugee Settlement. Journal of Consulting and 
Clinical Psychology, 72(4), 579-587. https://doi.org/10.1037/0022-006X.72.4.579.

Otto, M. W., \& Hinton, D. E. (2006). Modifying Exposure-Based CBT for Cambodian Refugees with Posttraumatic Stress Disorder. Cognitive and Behavioral Practice, 13(4), 261-270. https://doi.org/10.1016/j.cbpra.2006.04.007.

Palic, S., \& Elklit, A. (2011). Psychosocial Treatment of Posttraumatic Stress Disorder in Adult Refugees: A Systematic Review of Prospective Treatment Outcome Studies and a Critique. Journal of Affective Disorders, $137(1-3), 8-23$. https://doi.org/10.1016/j.jad.2010.07.005.

Schouler-Ocak, M., Reiske, S.-L., Rapp, M. a., \& Heinz, A. (2008). Cultural Factors in the Diagnosis and Treatment of Traumatised Migrant Patients from Turkey. Transcultural Psychiatry, 45(4), 652-670. https:// doi.org/10.1177/1363461508100787.

Tay, A. K., Rees, S., Chen, J., Kareth, M., \& Silove, D. (2015). The Structure of Post-Traumatic Stress Disorder and Complex Post-Traumatic Stress Disorder amongst West Papuan Refugees. BMC Psychiatry, 15(1), 111 . https://doi.org/10.1186/s12888-015-0480-3.

Wenk-Ansohn, M., \& Gurris, N. (2011). Intercultural Encounters in Counselling and Psychotherapy: Communication with the Help of Interpreters. Torture: Quarterly Journal on Rehabilitation of Torture Victims and Prevention of Torture, 21(3), 182-185. Retrieved from http://www.ncbi.nlm.nih.gov/PubMed/22057106.

Recebido em: 4/4/2018 Aprovado em: 24/2/2020 